\title{
Relationship between sleep quality and the level and pattern of BP
}

Yuli Huang, Weiyi Mai and Yunzhao Hu

We read Bakris' Year in Review article (Bakris, G. L. Hypertension in 2011: new insights-from risk factors to treatment implications. Nat. Rev. Cardiol. 9, 75-77 [2012] $)^{1}$ with great interest. We would like to discuss some views on the management of nondipping hypertension and the important relationship between sleep quality and blood pressure (BP).

The 'normal' circadian variation in $\mathrm{BP}$ is characterized by a $\geq 10 \%$ reduction in BP during the night. ${ }^{2}$ Individuals with this night-time dip in BP are known as 'dippers'. Patients with a nocturnal BP fall of $<10 \%$ are defined as 'nondippers.' ${ }^{2}$ Data indicate that the prevalence of a nondipping $\mathrm{BP}$ pattern might be as high as $~ 40-50 \%$. $^{3}$

Evidence suggests that nondippers are at greater risk of target organ damage than dippers. ${ }^{4}$ The potential reduction in cardiovascular risk associated with converting a nondipper into a dipper is still under debate. However, many researchers have sought ways to achieve this conversion, including via the prescription of chronotherapy with medications taken at night. In his Year in Review article, ${ }^{1}$ Bakris presented two studies that provided conflicting findings on the benefit of using chronotherapy to try to restore a dipping profile. More, similarly conflicting studies exist in the literature. For example, a randomized, controlled study of 115 patients $s^{5}$ and a registry study of $\sim 43,000$ individuals, ${ }^{3}$ respectively, showed that timing of antihypertensive treatment did and did not affect extent of dipping in BP. We believe that these inconsistent findings might result from differences in the pathophysiology and disease status of patients enrolled. Therefore, we believe that further investigation is needed to confirm the validity of the use of a chronotherapeutic approach for interventions aimed at restoring a dipping profile in hypertensive patients with differences in pathophysiology and disease status.
Quality of sleep has been reported to relate to the extent of nocturnal BP dipping in normotensive individuals, ${ }^{6}$ and poor sleep quality has been found to be significantly associated with nondipping hypertension. ${ }^{7,8}$ Our group has explored the associations between poor sleep quality, psychological stress, and nondipping BP. In 2011, we reported that poor sleep quality and high stress status are closely associated with increased activation of the sympathetic nervous system, and that they are independent predictors of nondipping hypertension. ${ }^{9}$ We also found that adding the mild sedative zolpidem to standard antihypertensive therapy is effective in improving sleep quality and stress status, and converting nondippers with poor sleep quality into dippers. ${ }^{10}$

In his Year in Review article, ${ }^{1}$ Bakris discussed a study by Fung et al. ${ }^{11}$ that demonstrated that decreased slow-wave sleep was associated with incident hypertension. Bakris indicated that physicians should be aware of decreased deep sleep as a risk factor for hypertension and ensure that patients get an extended rest period at night by decreasing stimuli such as caffeine and liquid intake. ${ }^{1}$ We think it would be interesting to explore the effect of sleep aids, such as zolpidem, on the incidence of hypertension in patients who have difficulty achieving good-quality sleep. Further understanding of the mechanisms by which poor sleep quality effects BP variation would be useful in directing future research and clinical management.

Department of Cardiology, the Affiliated Hospital at Shunde (the First People's Hospital of Shunde), Southern Medical University, Penglai Road 1, Daliang Town, Shunde District, Foshan 528300, China (Y. Huang, Y. Hu). Department of Cardiology, the First Affiliated Hospital of Sun Yat-sen University, 58 Zhongshan Road 2, Guangzhou 510080 , China (W. Mai).
Correspondence to: W. Mai wymai@hotmail.com

\section{Acknowledgments}

The authors of this manuscript are funded by the Scientific Research Fund of Guangdong, China (No: 2011B031800021); Cardiovascular Medicine Research Fund of Guangdong, China (No: 2009X20, 2011X38), and Medical Scientific Research Grant of Health Ministry of Guangdong province, China (No: B2011310).

\section{Competing interests}

The authors declare no competing interests.

1. Bakris, G. L. Hypertension in 2011: new insights - from risk factors to treatment implications. Nat. Rev. Cardiol. 9, 75-77 (2012).

2. Chobanian, A. V. et al. The seventh report of the Joint National Committee on Prevention, Detection, Evaluation, and Treatment of High Blood Pressure: the JNC 7 report. JAMA 289, 2560-2572 (2003).

3. de la Sierra, A. et al. Prevalence and factors associated with circadian blood pressure patterns in hypertensive patients. Hypertension 53, 466-472 (2009).

4. Birkenhager, A. M. \& van den Meiracker, A. H. Causes and consequences of a non-dipping blood pressure profile. Neth. J. Med. 65, 127-131 (2007).

5. Hermida, R. C. \& Ayala, D. E. Chronotherapy with the angiotensin-converting enzyme inhibitor ramipril in essential hypertension: improved blood pressure control with bedtime dosing. Hypertension 54, 40-46 (2009).

6. Loredo, J. S., Nelesen, R., Ancoli-Israel, S. \& Dimsdale, J. E. Sleep quality and blood pressure dipping in normal adults. Sleep 27, 1097-1103 (2004).

7. Yilmaz, M. B. et al. Sleep quality among relatively younger patients with initial diagnosis of hypertension: dippers versus non-dippers. Blood Press. 16, 101-105 (2007).

8. Erden, l. et al. Poor-quality sleep score is an independent predictor of nondipping hypertension. Blood Press. Monit. 15, 184-187 (2010).

9. Huang, Y. et al. Poor sleep quality, stress status, and sympathetic nervous system activation in nondipping hypertension. Blood Press. Monit. 16, 117-123 (2011).

10. Huang, Y. et al. The effect of zolpidem on sleep quality, stress status, and nondipping hypertension. Sleep Med. 13, 263-268 (2012).

11. Fung, M. M. et al. Decreased slow wave sleep increases risk of developing hypertension in elderly men. Hypertension 58, 596-603 (2011). 